\title{
Surgical Oncology Practice in the Wake of COVID-19 Crisis
}

\author{
Shailesh V. Shrikhande ${ }^{1} \cdot$ Adarsh Chaudhary $^{2} \cdot$ Aravind Krishnamurthy $^{3} \cdot$ G. V. Rao ${ }^{4} \cdot$ Harit Chaturvedi $^{5}$. \\ Madhabananda $\mathrm{Kar}^{6}$ • S. V. S. Deo ${ }^{7}$. Vikram Chaudhari ${ }^{8}$. S.P. Somashekhar ${ }^{9}$
}

Received: 11 June 2020 / Accepted: 21 September 2020 / Published online: 7 November 2020

(C) Indian Association of Surgical Oncology 2020

\begin{abstract}
The coronavirus disease 2019 (COVID-19) caused by the severe acute respiratory syndrome (SARS-CoV-2) outbreak has placed unprecedented challenges globally dismantling healthcare systems and forcing rapid transformations of healthcare services. In patients with cancer, these changes are having profound effects on vital aspects of their care. It has been advised that hospitals discontinue elective surgery and work on triage of nonemergent surgical procedures during the pandemic. The purpose of this article is to highlight the recommendations and adapted workflow from the private and public tertiary level hospitals in India advising on the best practices and views on better patient management, redesigning of SOPs for OR, surgeon, and staff safety and resumption of cancer care especially from surgical perspective. Different concerns are addressed that are necessary to optimize the quality of care provided to COVID-19 patients and to reduce the risk of viral transmission to other patients or healthcare workers.
\end{abstract}

Keywords Cancer care $\cdot$ COVID-19 $\cdot$ Oncology $\cdot$ SARS-CoV-2 $\cdot$ Surgical oncology

\section{Introduction}

The recent outbreak of novel coronavirus 2 (SARS-CoV-2)associated disease (COVID-19) has quickly gained pandemic status transforming healthcare delivery worldwide. Nationwide lockdowns and prioritization of healthcare for

Shailesh V. Shrikhande

shrikhandesv@tmc.gov.in

1 Tata Memorial Hospital, Tata Memorial Centre, Homi Bhabha National Institute, Mumbai, Maharashtra, India

2 Institute of Digestive and Hepatobiliary Sciences, Medanta-The Medcity, Delhi, India

3 Department of Surgical Oncology, Adyar Cancer Institute, Chennai, Tamilnadu, India

4 Asian Institute of Gastroenterology, Hyderabad, Telangana, India

5 Max Institute of Cancer Care, Max Group of Hospitals, Delhi, India

6 AIIMS, Bhubaneshwar, Orissa, India

7 Department of Surgical Oncology, AIIMS (ICRH and NCI), Delhi, India

8 Department of Surgical Oncology, Tata Memorial Hospital, Tata Memorial Centre, Homi Bhabha National Institute, Mumbai, Maharashtra, India

9 Manipal Hospitals, Bengaluru, Karnataka, India acute care have disrupted and delayed the delivery of care to patients with cancer. It is reported that patients with cancer are already at an increased risk of infection [1]. Cancer patients who contract COVID-19 have more than triple the risk for severe health events including admission to the intensive care unit for invasive ventilation and rapid clinical deterioration [2]. An Italian study assessing the case fatality of COVID19 reported that $72(20.3 \%)$ among 355 patients who died had active cancer [3].

As the COVID-19 pandemic deepens and widens, hospitals are bracing up for challenging times as the crisis significantly reduced the outpatient department (OPD), hospital inpatient care (IPD), and operating room (OR) footfalls. The oncology community is striving profoundly to reorganize the oncological care in order to reduce in-person clinical appointments, prioritizing treatment strategies, educational activities, and staff management and rescheduling cancer surgeries without compromising cancer outcomes. It has been advised that hospitals discontinue elective surgery and work on triage of nonemergent surgical procedures during the pandemic [4]. On the contrary, in the absence of treatment, cancer may spread or develop resistance to treatments causing multiple complications or, in some instances, death. We may see a drop in the proportion of early detected malignancies due to delayed presentation which could have cascading effects on cancer patients. Hence, it is imperative to design and implement 


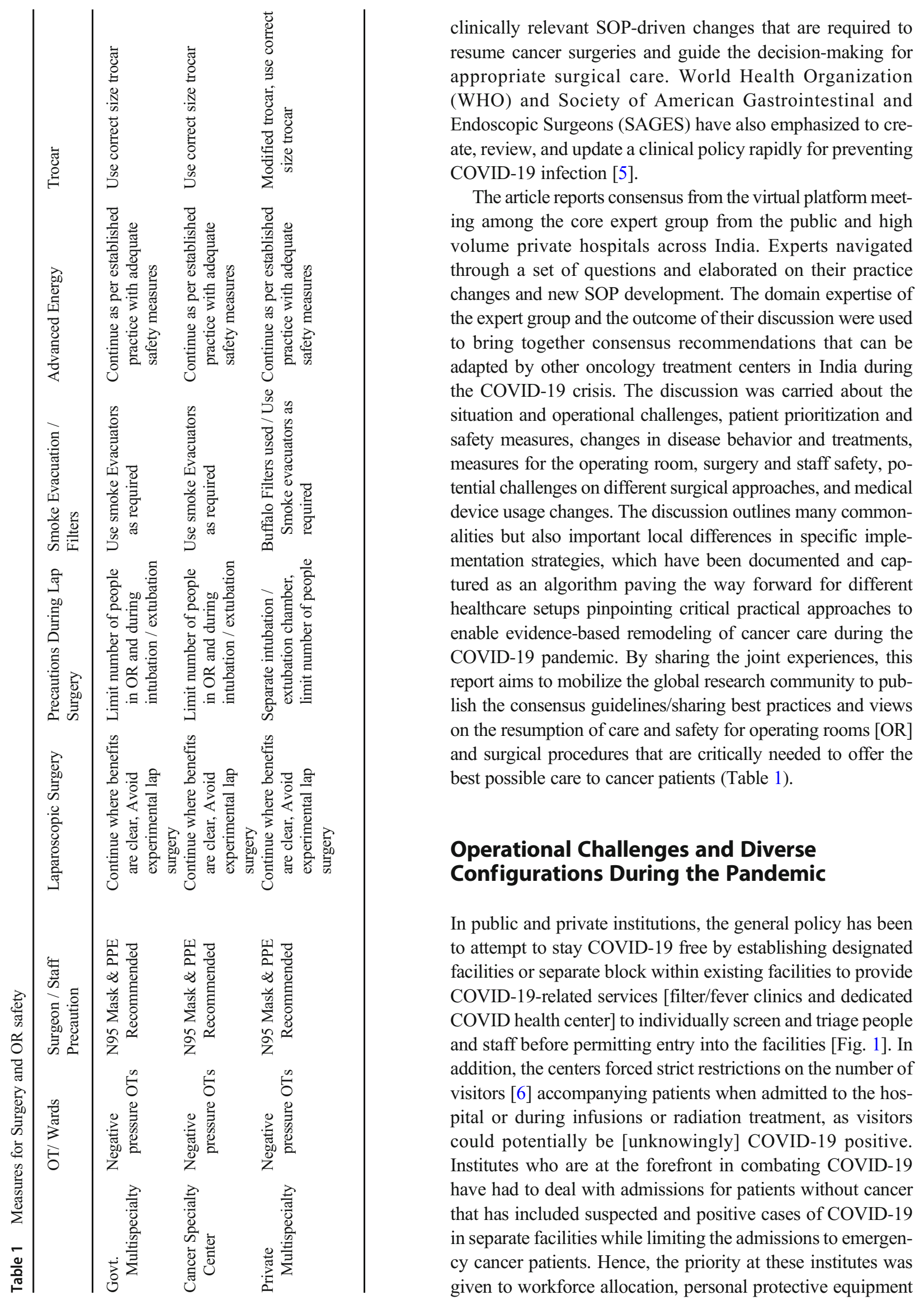


Fig. 1 Triage strategy in patients with cancer

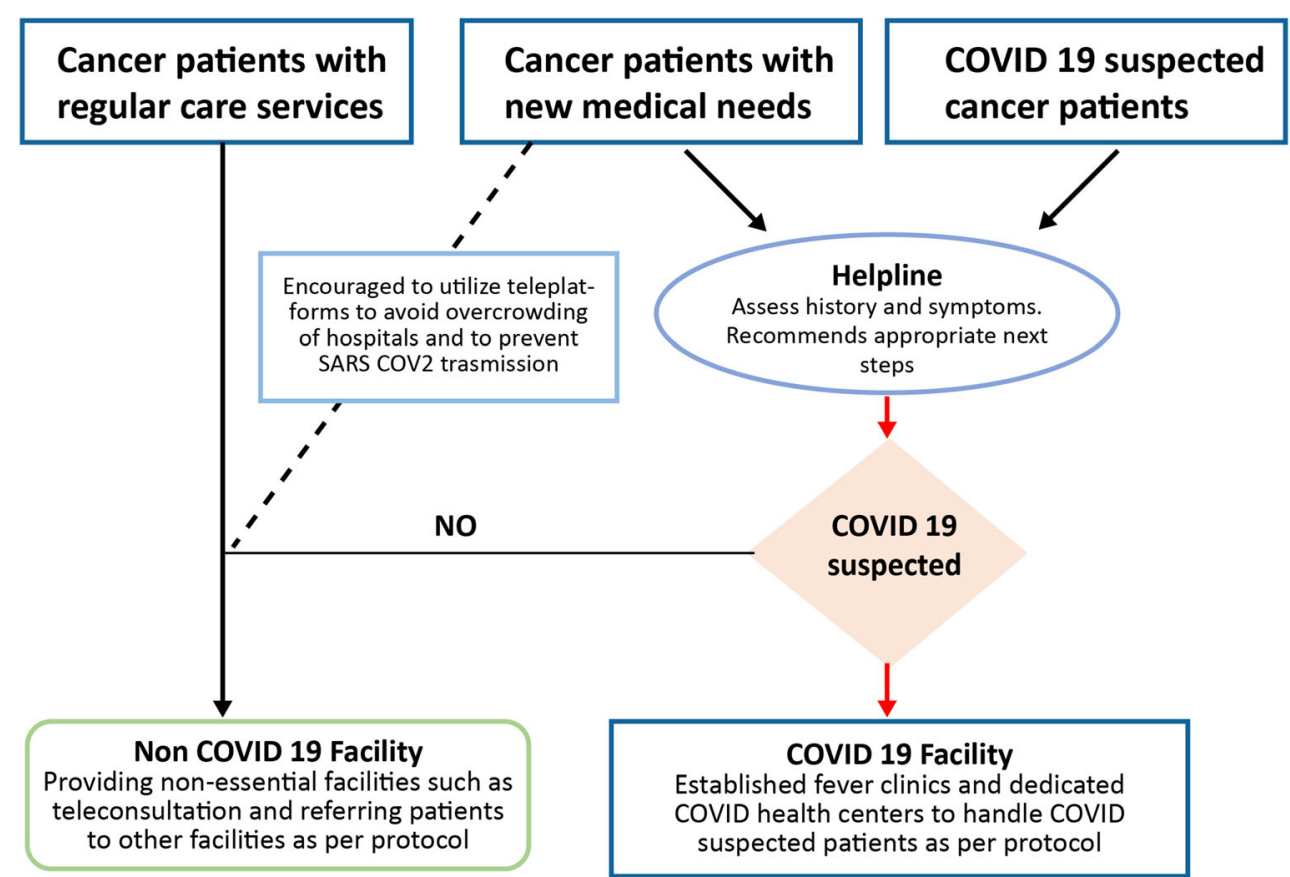

[PPE] acquisition, and creation of capacity for ICU's and ventilators. Given the high transmissibility rate of SARS-Cov-2, all centers have facilitated face-to-face consultations, whenever possible, taking place via web consulting or by telephone calls to avoid overcrowding of hospitals and to ensure patients are not exposed to SARS-CoV-2 virus during travel or in health facilities. For survivor groups, online meetings have been organized through teleconferencing software platforms. There were no specific changes in treatment and noted changes in disease behavior observed during the pandemic phase. The crisis is said to affect the clinical outcomes adversely due to delayed presentation and prolonged time before treatment initiation. No significant increase in hospital length of stay has been reported except in a few cases.

\section{Patient Prioritization System for Cancer Patients}

Priority setting for the delivery of cancer therapies in the context of a pandemic will be strongly influenced by several factors, including the patient's location and resource allocation-from protective gear, hospital beds, and to nursing and doctor time. Few guidelines are currently available for different surgical considerations and prioritization [7, 8]; however, the prioritization process should be adjustable to local, regional, and national epidemiological trends and changes in COVID-19 care. The prioritization process should also take a facility's resources, priorities, and patient needs into account [9]. The prioritization process, principles, and framework should be transparent to hospitals, healthcare workers, and the public. The benefits of transparency include reducing ethical dilemmas. There are multiple considerations in developing the prioritization process, including a list of canceled and delayed procedures, a strategy for a phased opening of ORs, PPE availability, and issues related to increased OR volume. Priority allocation of resources and staff to COVID-19 care, a continuation of emergency, semi-urgent essential care, and gradual resumption of withheld elective clinical activities need to be balanced and should now continue side by side considering that the COVID-19 pandemic is here to stay for a longer period. Though this would be guided by local COVID-19 situation and the extent to which hospital infrastructure is occupied by COVID-19 patients, it is gradually being realized that delays in non-COVID-19 care can be equally if not more devastating and this cannot be postponed indefinitely [10].

Figure 2 depicts prioritization (Priority A has highest and $\mathrm{C}$ being lowest) considerations based on factors like the chances of cure, availability of effective non-surgical treatment options or effective of upfront non-surgical treatments, and risk for progression.

\section{Measures for Patient Safety}

COVID-19 appears to affect people of all ages; however, cancer patients who are usually characterized by lower immunity and immuno-depressed state because of their chemotherapy or surgery are at higher risk for serious medical complications and increased mortality once infected with COVID-19 compared with the healthy population [2]. Hence, more attention should be paid to cancer patients as the effective prevention of cross-infection of COVID-19 and the rational arrangement of 
Fig. 2 Patient prioritization flow (A to $\mathrm{C}$-highest to lowest)

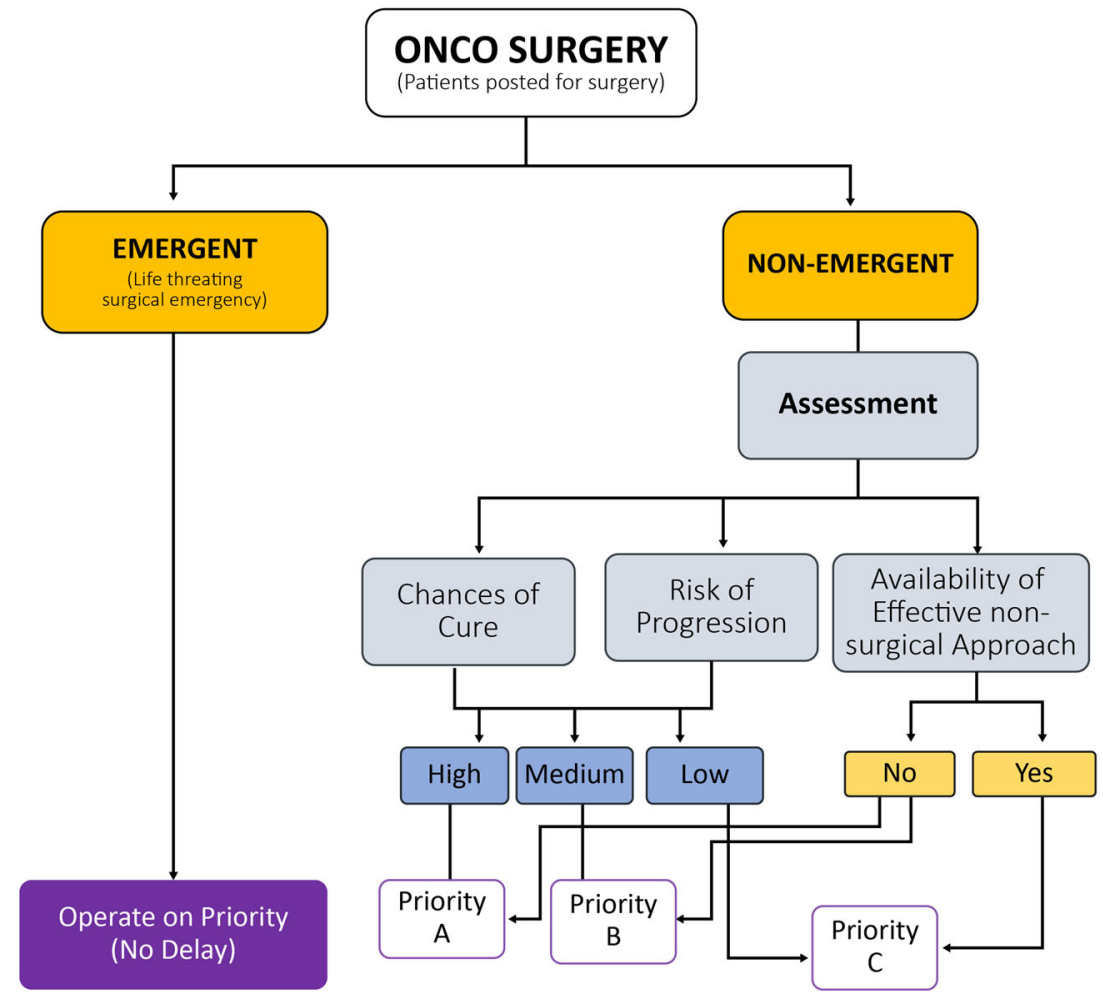

anticancer treatment are extremely important. Several mandatory measures are being carried out in hospitals during this period to alleviate the difficulties of patients with cancer in seeking medical aid and simultaneously avoiding COVID-19related cross-infection between patients and medical staff.

First, SOPS on safety measures, a proper infection control protocol, SOPS for the OTs, and the postoperative wards have been created. Mandatory routine tests for the symptoms of SARS-CoV-2 are being performed in all patients and staff in order to reduce the risk of in-hospital transmission of COVID-19.

Second, multiple on-site temperature tests are performed in the triage tent at the entrances of the hospital authorized by a multidisciplinary triage team. Strict screening strategy with a standard questionnaire scoring system designed by ICMR has been implemented for all patients, visitors, and medical staff presenting to the hospital. Patients who do not pass the scoring track are restricted to enter OPD and were dealt separately in the clinics outside. Furthermore, some hospital initiatives such as code green have been taken to restrict the free movement of high-risk patients. These reported safety measures may be of great value to help guide patients with cancer smoothly and safely through the crisis.

\section{Measures for Operating Room, Surgery, and Staff Safety}

The overarching goal in cancer care during the COVID-19 crisis is to provide compassionate and high-quality cancer care while continuing to look after patient and staff safety. SARS-CoV-2 is considered most contagious when the patient is symptomatic, and the evidence suggests that it is transmitted by asymptomatic individuals as well [11]. Transmission before the onset of symptoms has been reported $[12,13]$ and has contributed to spreading among residents of a nursing facility in Washington, USA [14]. Transmission of SARS-CoV-2 within healthcare facilities to healthcare workers has been documented in China where 3.8\% of COVID-19 cases were reported in healthcare workers leading to five deaths [15]. Italy reported 15,314 infections among healthcare workers, representing $11 \%$ of all infections as of 10 April 2020 [16]. The World Health Organization reported 22,073 cases of COVID-19 among healthcare workers from 52 countries as of 8 April 2020 [17]; however, due to lack of systematic reporting, the actual number is underrepresented.

Due to the lockdown imposed by the government responding to this crisis, public and private institutes developed the outbreak response administrative measures such as modification of workflow and processes, management, coordination and movement of staff, the introduction of personal protective equipment for staff, and strengthening of the screening tests. Institutes formulated self-institutional guidelines for safety protocols based on the ASA and APSF, SSO, IASO, and AMASI guidelines. To deliver universal high-quality care through multidisciplinary medical guidance to cancer patients, virtual tumor boards were launched and department meetings have been initiated. One important consideration even in routine work is that all ORs should consider all patients as COVID-19 positive and must be accommodated in negative pressure operation rooms based on 
availability. Other measures include minimizing patient-provider encounters.

When compared with the flow of surgeries with preCOVID-19 phase, public institutes reported a 50-60\% drop in numbers of oncosurgeries but reported no change in emergency surgeries. To cope with the anticipated increased influx of COVID-19 patients, private institutes decided to continue with only emergency surgeries as normal during phase 1 lockdown. All priority oncosurgeries in private institutes were resumed back during the phase 2 lockdown and were made possible due to the development of a few internal policy changes specified above. There is an agreement that managing rotation of staff is a key issue in resuming services and minimizing exposure. One such strategy adopted was 2 weeks on and 2 weeks off considering the incubation period can be as long as 14 days. Another strategy adopted at private institutes suggests 3 days on and 3 days off duty for medical and radiation oncologists, encouraging every clinician to visit alternate day for 2-3 h.

Perioperative COVID-19-positive status confers a very high risk of perioperative pulmonary complications and mortality. Risk is higher for patients undergoing major cancer surgery [18]. Many guidelines strongly recommend testing for SARS-COV-2 prior to the operation [7] and emphasized on RT PCR testing to all patients. This can be resource exhaustive and may not be completely practical at this moment for universal application considering the limited number of test kit availability and rising incidence across the country. Also, RT PCR is only $70 \%$ sensitive, and the risk of false negativity is significant [19]. Whether universal screening can be mandated for every patient undergoing surgery based on the available resources is yet unanswered. This is currently being governed by recommendations released from time to time by authorized national and local government health agencies. The process however is dynamic, and we will probably see routine preoperative testing to for COVID-19 in times to come considering the risk to the patient and healthcare workers both in absence of definitive treatment and immunity.

\section{Different Surgical Approaches and Potential Challenges}

Cancer surgery and other surgeries that cannot be avoided should be continued with adequate precautions as far as possible, and surgical approaches should be selected judiciously. A small but definite risk of transmission exists irrespective of the surgical approach [open, laparoscopic, or robotic]; however, one needs to take all the possible precautions to minimize it. It is well established that laparoscopy has not only a favorable impact on respiratory function but also less pain and reduced length of stay for patients. However, safety precautions are necessary when performing laparoscopy due to the risk of exposure and infection to the staff personnel [20]. Many emergency laparoscopic procedures will still be required during this pandemic, yet very little is known regarding the uptake of these procedures during the COVID-19 crisis surrounding SARS-CoV-2 virus transmission. There are concerns relating to COVID-19 transmission arising from the potential generation of SARS-CoV-2-contaminated aerosols from $\mathrm{CO}_{2}$ leakage during laparoscopic surgery, although there are no data to support it. Intubation and extubation remain one of the highest risk aerosol-generating procedures due to the high viral load in respiratory secretions [21]. However, performing minimally invasive robotic and laparoscopic surgery is not contraindicated if provided with certain modifications. Few hospitals have modified all the trocars and cannula with an external evacuator which goes through an undersealed double chamber sodium hypochlorite and the third chamber with a buffalo filter.

Laparoscopy allows for a self-contained operative field with less and possibly no spillage of fluids and tissues, thus minimizing risk to the operative staff. All institutes agreed that laparoscopic surgery can be continued where clear benefits have been evident but emphasized avoiding experimental laparoscopy during the current crisis. On the other hand, at some hospitals, all minimally invasive surgeries were suspended initially but have resumed especially in patients with prostate and lower rectum cancers where the benefit over open surgery is well established [22]. The temporary suspension was observed at institutes due to available infrastructure, patient number, surgery time, and staff availability. It is advisable to evaluate risk of COVID-19 in patients before any surgery, rather its high time to emphasize and follow infection control with the utmost care.

\section{Medical Device Usage and Adopting to Changes}

As laparoscopy is known to result in the earlier discharge of patients from hospitals and less dealing with surgical wounds and surgical site infections (SSIs), few guidelines have been established providing recommendations regarding laparoscopic surgical response to COVID-19 [23]. It is reported that the aerosol can contaminate personnel and all the furniture and surfaces in the room via airborne particles when released during laparoscopic surgery [leaks] or after the operation (exsufflation) [24]. Smoke evacuators used at the time of laparoscopy offer the unique advantage of being able to reduce the surgical plume in the abdominal cavity [20]. However, there is no compelling data to support the notion that COVID-19-a novel virus - is transmitted through the surgical plume or aerosolized laparoscopic gas. To disseminate knowledge and provide guidelines for minimally invasive 
surgery procedures, a recent study suggested the usage of lowpressure peritoneum, the use of balloon trocars, evacuating all pneumoperitoneum before trocar removal, or specimen extractions [25]. On emphasizing the importance of using smoke evacuators, hospitals should consider the use of new modalities such as suction devices placed near the electrosurgical site, to further decrease the aerosolization that could improve smoke evacuation beyond the simple vacuum and filter system [26]. However, there are no claims reporting any changes in surgical technique practices or use of any surgical devices including usage of energy devices, but adequate safety precautions must be taken.

\section{Patient Counseling, Teleconsultations, and Legal Implications}

Temporary halt on non-COVID-19 healthcare services has caused anxiety and confusion among cancer patients [27]. It is possible that the stress related to this pandemic may exacerbate other treatment-related effects and the psychosocial impact of the pandemic may disproportionately affect the cancer patients. It is important that clinicians directly acknowledge patients' concerns about their individual risks and anxieties. To address these psychosocial needs of the patients, institutions have started providing psychosocial counseling not just to patients but also to the staff personnel for empowerment and control during this pandemic.

During the COVID-19 crisis, physicians are providing competent care by placing patient welfare above other interests, catering the information to enable patients to make wellconsidered decisions and promoting continuity of care, while respecting patient privacy and confidentiality. Hospitals implemented teleconsultations to patients by strictly adhering to the requirements of Telemedicine Practice Guidelines published by the Indian government [28]. Few hospitals have integrated a teleconsultation software with the institutes HIS system, and a separate teleconsultation team is established to provide personalized telehealth advice electronically. Patients were encouraged to consent for teleconsultation, and identification was confirmed through a government ID while providing safeguards to protect the patient's privacy and security and documenting the prescription and clinical evaluation.

Informed consenting is a fundamental step in clinical practice. A small early study from China suggested that cancer patients who underwent surgery or chemotherapy in the month preceding the appearance of the virus had a higher risk (75\%) of developing a severe episode compared with those who had not undergone surgery or chemotherapy (43\%) [2]. Further evidence described the high mortality rate and poorer outcomes associated with COVID-19 in cancer patients with older age $[18,29]$. Hence, it is crucial to include the data about the effect of cancer and cancer treatment risks in the wake of the COVID-19 crisis and communicated in a clear and detailed manner to the patients during the informed consenting process by the treating oncologist. All institutes involved in this discussion have already adapted to this measure to support individual autonomy to patients about their treatment decisions.

\section{Conclusion}

In the wake of the COVID-19 pandemic, we recommend rigorous preparations in terms of internal administrative measures. These measures include the modification of infrastructure and processes, management of staff and patients, strict screening and triage strategies, establishment and frequent updating of protocols, and clinical recommendations and infection prevention strategies. Appointments of patients on follow-up should be managed through telemedicine or web consulting to reduce the possibility of hospital visits and eliminating the risk of spreading the virus. All treatment decisionmaking should follow standard guidelines and must be taken in the context of a multidisciplinary tumor board. The informed consenting process must include recent data about the risk and benefits of treatment in the context of the specific COVID-19 pandemic status.

Data Availability NA

\section{Compliance with Ethical Standards}

Conflict of Interest The authors declare that they have no conflict of interest.

Ethics Approval NA

Consent to Participate NA

Consent for Publication NA

Code Availability NA

\section{References}

1. Infections in people with cancer. American Cancer Society. https:// www.cancer.org/treatment/treatments-and-side-effects/physicalside-effects/low-blood-counts/infections.html. Accessed 15 May 2020

2. Liang W, Guan W, Chen R, Wang W, Li J, Xu K, Li C, Ai Q, Lu W, Liang H, Li S, He J (2020) Cancer patients in SARS-CoV-2 infection: a nationwide analysis in China. Lancet Oncol 21:335337

3. Onder G, Rezza G, Brusaferro S (2020) Case-fatality rate and characteristics of patients dying in relation to COVID-19 in Italy. JAMA 323(18):1775-1776 
4. COVID 19: Elective Case Triage Guidelines for Surgical Care. https://www.facs.org/-/media/files/covid19/guidance_for_triage of nonemergent_surgical_procedures.ashx. Accessed $0 \overline{1}$ June 2020

5. World Health Organization (2020) World Health Organization coronavirus disease (COVID-19) outbreak: rights, roles and responsibilities of health workers, including key considerations for occupational safety and health. https://www.who.int/docs/default-source/ coronaviruse/who-rights-roles-respon-hw-covid-19.pdf?sfvrsn= bcabd401 0. Accessed 05 May 2020

6. World Health Organization (2020) Infection prevention and control of epidemic- and pandemic-prone acute respiratory diseases in healthcare. https://apps.who.int/iris/bitstream/handle/10665/ $112656 / 9789241507134$ eng.pdf; jsessionid= 6E0292B2C7D5F2DDB7645EA95416A70C? sequence $=1$. Accessed 30 April 2020

7. Sages recommendations regarding surgical management of gastric cancer patients during the response to the COVID19 crisis. https:// www.sages.org/sages-recommendations-surgical-managementgastric-cancer-covid-19-crisis/. Accessed 30 April 2020

8. American college of surgeons (2020) Recommendations for prioritization, treatment and triage of breast cancer patients during the COVID-19. Pandemic: Executive Summary. https://www.facs.org/ quality-programs/cancer/executive-summary. Accessed 30 April 2020

9. Krishnamurthy A, Gopinath KS (2020) The need for prioritizing cancer surgeries amidst the COVID-19 pandemic. Indian J Surg Oncol 11(2):169-170

10. van de Haar J, Hoes LR, Coles CE, Seamon K, Fröhling S, Jäger D, Valenza F, de Braud F, de Petris L, Bergh J, Ernberg I, Besse B, Barlesi F, Garralda E, Piris-Giménez A, Baumann M, Apolone G, Soria JC, Tabernero J, Caldas C, Voest EE (2020) Caring for patients with cancer in the COVID-19 era. Nat Med 26:665-671

11. Rothe C, Schunk M, Sothmann P, Bretzel G, Froeschl G, Wallrauch C, Zimmer T, Thiel V, Janke C, Guggemos W, Seilmaier M, Drosten C, Vollmar P, Zwirglmaier K, Zange S, Wölfel R, Hoelscher M (2020) Transmission of 2019-nCoV infection from an asymptomatic contact in Germany. N Engl J Med 382:970-971

12. Tong Z, Tang A, Li K, Li P, Wang HL, Yi JP, Zhang YL, Yan JB (2020) Potential presymptomatic transmission of SARS-CoV-2, Zhejiang Province, China, 2020. Emerg Infect Dis 26:1052-1054

13. Ye F, Xu S, Rong Z, Xu R, Liu X, Deng P, Liu H, Xu X (2020) Delivery of infection from asymptomatic carriers of COVID-19 in a familial cluster. Int J Infect Dis 94:133-138

14. Kimball A (2020) Asymptomatic and presymptomatic SARS-CoV2 infections in residents of a long-term care skilled nursing facility-King County, Washington, march 2020. Morb Mortal Wkly Rep 69:377-381

15. The Novel Coronavirus Pneumonia Emergency Response Epidemiology Team. The epidemiological characteristics of an outbreak of 2019 novel coronavirus diseases (COVID-19) - China, 2020. http://weekly.chinacdc.cn/en/article/id/e53946e2-c6c441e9-9a9b-fea8db1a8f51. Accessed 05 May 2020
16. Integrated surveillance of COVID-19 in Italy. https://www. epicentro.iss.it/en/coronavirus/bollettino/Infografica_10aprile\% 20ENG.pdf. Accessed 10 May 2020

17. Coronavirus disease 2019 (COVID-19): situation report (2020) World Health Organization: 82. https:/www.who.int/docs/defaultsource/coronaviruse/situation-reports/20200411-sitrep-82-covid19.pdf?sfvrsn=74a5d15_2. Accessed 10 May 2020

18. Nepogodiev D, Glasbey JC, Li E, Omar OM, Simoes JF, Abbott TE, Cunha MF (2020) Mortality and pulmonary complications in patients undergoing surgery with perioperative SARS-CoV-2 infection: an international cohort study. Lancet:1-1. https://doi.org/10. 1016/S0140-6736(20)31182-X

19. Interpreting a covid-19 test result. https://www.bmj.com/content/ 369/bmj.m1808. Accessed 01 June 2020

20. SAGES and EAES recommendations regarding surgical response to covid-19 crisis. https://www.sages.org/recommendationssurgical-response-covid-19/. Accessed 15 May 2020

21. Meng L, Qiu H, Wan L, Ai Y, Xue Z, Guo Q, Deshpande R, Zhang L, Meng J, Tong C, Liu H, Xiong L (2020) Intubation and ventilation amid the COVID-19 outbreak Wuhan's experience. Anesthesiology 132(6):1317-1332

22. Cheong C, Kim NK (2017) Minimally invasive surgery for rectal cancer: current status and future perspectives. Indian J Surg Oncol 8:591-599

23. Society of American Gastrointestinal and Endoscopic Surgeons (SAGES) (2020) Notes from the battlefield. https://www.sages. org/notes-fromthe-battlefield-march-30-2020. Accessed 15 May 2020

24. Tuech JJ, Gangloff A, Di Fiore F et al (2020) Strategy for the practice of digestive and oncological surgery during the Covid-19 epidemic. J Visc Surg 157:S7-S12. https://doi.org/10.1016/j. jviscsurg.2020.03.008

25. Zheng MH, Boni L, Fingerhut A (2020) Minimally invasive surgery and the novel coronavirus outbreak: lessons learned in China and Italy. Ann Surg 272:e5-e6. https://doi.org/10.1097/SLA. 0000000000003924

26. Schultz L (2015) Can efficient smoke evacuation limit aerosolization of bacteria? AORN J 102:7-14

27. Dalal NV (2020) Social issues faced by cancer patients during the coronavirus (COVID-19) pandemic. Cancer Res Stat Treat 3:141

28. Telemedicine Practice Guidelines. Medical council of India. https:// www.mohfw.gov.in/pdf/Telemedicine.pdf. Accessed 15 May 2020

29. Wang D, Hu B, Hu C, Zhu F, Liu X, Zhang J, Wang B, Xiang H, Cheng Z, Xiong Y, Zhao Y, Li Y, Wang X, Peng Z (2020) Clinical characteristics of 138 hospitalized patients with 2019 novel coronavirus-infected pneumonia in Wuhan, China. JAMA 323: 1061-1069

Publisher's Note Springer Nature remains neutral with regard to jurisdictional claims in published maps and institutional affiliations. 